\title{
Arthritis in the highlands of Papua New Guinea
}

Kevin D Pile, John E Richens, Rodger M Laurent, Kuldeep Bhatia, Madhuri L Prasad, Tony Lupiwa, Bernard J Hudson, John Tapsall, Rosemary McPetrie

\section{Abstract}

Acute polyarthritis is an important cause of morbidity in many tropical countries. Classification has often been difficult, with the term tropical polyarthritis used for those in whom a diagnosis could not be made. The implication that this is a distinct entity is probably incorrect, with likely causes being septic arthritis or post-infective reactive arthritis.

This study aimed to determine the types of arthritis found in 43 patients ( 30 men) presenting consecutively to the Goroka Base Hospital in the Eastern Highlands of Papua New Guinea. Gonococcal arthritis was diagnosed in eight patients (six men) on the basis of isolation of Neisseria gonorrhoeae from the joint aspirate. In all cases the $\boldsymbol{N}$ gonorrhoeae was identified by the closed culture system on chocolate agar, but not always by routine plating. There were no specific clinical features that identified patients with a gonococcal septic arthritis.

The remaining 34 patients had an undifferentiated oligoarthritis. The pattern of arthritis in men and women was of a lower limb pauciarticular arthritis with a predilection for the knee and ankle joints. A total of $30 \%$ of male patients had a history of urethral discharge and $44 \%$ of all patients had preceding diarrhoea. Arthritis was the only feature in $59 \%$ of patients and in $32 \%$ there was an associated enthesitis.

In this study most patients had an oligoarthritis consistent with a reactive arthritis or a septic arthritis due to $\boldsymbol{N}$ gonorrhoeae. Broth inoculation of synovial fluid was the best method to isolate $\mathbf{N}$ gonorrhoeae, with standard methods for gonococcal isolation failing in some patients. It is recommended that the term 'tropical polyarthritis' is no longer used as it does not refer to a specific entity but consists of several known arthritides.

(Ann Rheum Dis 1993; 52: 49-53)

Acute polyarthritis is an important cause of morbidity in many tropical countries. ${ }^{1}$ Its classification has been difficult, with the term tropical polyarthritis used for those in whom a diagnosis cannot be made. The implication that this is a distinct entity is probably incorrect, with likely causes being septic arthritis or post-infective reactive arthritis. ${ }^{1}$

A distinct non-suppurative arthritis was originally described from the Papua New Guinea highlands in 1969. An incomplete Reiter's syndrome was thought unlikely in the absence of mucocutaneous features and a higher than expected incidence in women. ${ }^{2}$ This was in contrast with an earlier report from the coastal Port Moresby hospital in which Reiter's disease or possible Reiter's disease accounted for half of the admissions for arthritis. ${ }^{3}$

Subsequent reports have been contradictory, considering either septic arthritis or reactive arthritis as the most common cause. ${ }^{4-6}$ Reasons for these differences include different racial groups, the geographical area studied, details of the clinical examination, and available microbiological facilities.

The aim of this study was to determine the types of arthritis in patients presenting with musculoskeletal symptoms in the highlands of Papua New Guinea. The most common types of arthritis were an oligoarthritis consistent with reactive arthritis, and septic arthritis due to Neisseria gonorrhoeae.

\section{Patients and methods}

PATIENTS

There were 43 patients presenting with musculoskeletal symptoms to Goroka Base Hospital, the provincial hospital for the Eastern Highlands Province of Papua New Guinea, during August and September 1989. They were consecutive patients attending medical outpatients, casualty services, or requiring admission.

Goroka Base Hospital is the major referral hospital for the Eastern Highlands Province. Transport by air or road from rural health centres throughout the Eastern Highlands Province is freely available to all inhabitants. A nominal fee may be charged for outpatient or inpatient medical care, but the population generally is not poor, and no person is ever refused treatment.

All patients were from the Eastern Highlands Province and were of Melanesian origin. Their occupations were predominantly rural, which includes subsistence farming and coffee growing.

Previous studies of this population have not shown arbovirus, especially Ross River virus, infection to be a cause of arthritis in these subjects. $^{2}{ }^{5-7}$ We therefore decided to carry out a study over a shorter time period.

Controls for HLA typing were matched for age, sex, and geographical origin; controls for serological testing were matched for age, sex, and place of residence. Approval for this study was given by the Papua New Guinea Medical Research Advisory Committee. 
METHODS

A history and examination of each patient was performed by a rheumatologist (KDP).

Synovial fluid was available from 24 patients. Total cell count, differential cell count, and examination for crystals using compensated polarised light microscopy were undertaken. The culture of synovial fluid was by three methods: (a) 5-10 ml synovial fluid was inoculated at the bedside into Liquoid TSB Roche (Hoffman-LaRoche Diagnostica Basle, Switzerland) blood culture bottles prewarmed to $30-37^{\circ} \mathrm{C}$. These contained tryptic soy broth in a closed system with a single dip slide that incorporated three separate agar slopes (chocolate, MacConkey, malt). They were incubated at $37^{\circ} \mathrm{C}$ for 10 days with daily inversion of the bottles to flood the dip slide agars. Growing colonies were taken for Gram staining. Gram negative diplococci were subcultured onto chocolate agar and gonococcal isolation media consisting of GC agar base (Difco, Detroit, MI, USA), $9 \%$ saponin lysed horse blood plus VCN inhibitor (Becton Dickinson, Cockeysville, MD, USA). Other organisms were subcultured onto chocolate agar and 5\% horse blood agar aerobically and anaerobically. All aerobic plates were incubated at $37^{\circ} \mathrm{C}$ in a $5 \% \mathrm{CO}_{2}$ atmosphere for 48 hours. Synovial fluid was inoculated in the laboratory within 10 minutes of collection directly onto chocolate agar, gonococcal isolation media, and horse blood agar and incubated as described. Spun deposits of synovial fluid were also processed with Gram stain and inoculation of chocolate and 5\% horse blood agar plates incubated as described. Identification of isolates and antibiotic sensitivity testing were performed according to the methods of the Australian Gonococcal Surveillance Programme. ${ }^{89}$ Four gonococcal isolates were auxotyped and serotyped by the coordinating laboratory of the Australian Gonococcal Surveillance Programme at the Prince of Wales Hospital (Sydney, Australia). Auxotyping was by the method of La Scolea and Young. ${ }^{10}$ Serotyping was with a set of 12 monoclonal antibodies to gonococcal outer membrane epitopes, prepared by Syva (Palo Alto, CA, USA).

Peripheral blood was assessed by complete blood count, erythrocyte sedimentation rate (ESR; Wintrobe), and evidence of microfilaraemia by nucleopore filtration of peripheral blood. " Antinuclear antibodies were measured by indirect immunofluoresence on HEp2 substrate (Kallestad Laboratories, TX, USA). IgM and IgA rheumatoid factors were measured by an enzyme linked immunosorbent assay (ELISA; Pharmacia Diagnostics, Uppsala, Sweden). Titres of antibodies to streptolysin O (Wellcome Diagnostics, Dartford, United Kingdom) and deoxyribonuclease B (Streptonase-B, Wampole Laboratories, NJ, USA) were determined by tube testing. Ross River serology specific for IgM antibodies was tested by ELISA (PanBio, Queensland, Australia) and confirmed with an IgM antibody class capture ELISA. ${ }^{12}$ Human leucocyte antigen typing was performed on 41 patients. ${ }^{13}$ There was an equal number of controls tested and they were matched to the patients for age, sex, and geographical origin.
Results

PATIENTS

Forty three patients were studied during the seven week period: 30 men, median age 25 years, interquartile range 19-32 years, and 13 women, median age 23 years, interquartile range $18-36$ years.

Table 1 gives the main types of arthritis.

\section{SEPTIC ARTHRITIS}

Gonococcal arthritis was diagnosed in eight patients (six men, two women) on the basis of isolation and growth of $N$ gonorrhoeae from the joint aspirates. Gram negative diplococci were identified by Gram staining on the initial aspirate in only one patient. The remainder were all identified in the closed blood culture system on chocolate agar after a mean of 4.5 days (range 2-10 days). Routine plates were discarded at 48 hours and therefore did not identify five of the eight positive patients using the closed blood culture system.

The median duration of symptoms in this group was 32 days, with episodic symptoms occurring in joints which were clinically unaffected at the time of presentation to hospital. A monarthritis was present in half the patients, the median number of joints affected being $2 \cdot 0$ (range one to eight). The knee was affected in six patients, the wrist in three, metatarsophalangeal joints in two, and one each of elbow, ankle, subtalar joint, acromioclavicular, and sternoclavicular joints. Only one joint was aspirated for each patient. Four also had either rotator cuff tendinitis or trochanteric bursitis/ enthesitis, and one tenosynovitis of the flexor tendons of the hands. Prominent epitrochlear lymphadenopathy was associated with gonococcal arthritis of the wrist and elbow. No skin lesions were identified in this group of patients.

Peripheral blood white cell counts were within the normal range in all patients with identified gonococcal septic arthritis and the median ESR was $33 \mathrm{~mm} /$ hour (interquartile range 6-51). The median synovial fluid white cell count in patients with gonococcal arthritis was $28 \times 10^{9} / 1$ (range $1 \cdot 2-35 \times 10^{9} / 1$ ) with a median polymorphonuclear leucocyte differential of $84 \%$.

Four of the isolates were penicillinase producing $N$ gonorrhoeae (PPNG). Two of these isolates, and two non-PPNG strains were transported to the Australian Gonococcal Surveillance Programme for auxotyping, serotyping, and full antibiotic sensitivity testing. All were IA2 serotype, three were proline, serine, isoleucine requirers, and one was a proline requiring strain. Of the non-PPNG isolates, the minimum inhibitory concentration (MIC) for penicillin was $0 \cdot 125 \mathrm{mg} / \mathrm{l}$ in both instances. There was no high level resistance to either tetracycline or chloramphenicol and the MIC

Table 1 Types of arthritis found in 43 patients

\begin{tabular}{lc}
\hline Type of arthritis & Number (\%) of patients \\
\hline Gonococcal septic arthritis & $8(19)$ \\
Gout & $1(2)$ \\
Undifferentiated oligoarthritis & $34(79)$ \\
\hline
\end{tabular}


was $2 \mathrm{mg} / \mathrm{l}$ or less in all instances. All strains were fully sensitive to ceftriaxone (MIC $<0.004$ $\mathrm{mg} / \mathrm{l})$, kanamycin $(\mathrm{MIC}<5 \mathrm{mg} / \mathrm{l})$, ciprofloxacin (MIC <0.008 mg/l), and spectinomycin (MIC $<20 \mathrm{mg} / \mathrm{l})$.

Treatment was with penicillin or chloramphenicol, the latter often being given after a poor response to penicillin. The appropriate antibiotic treatment resulted in a marked clinical improvement within one to two days.

GOUT

One patient had acute gout in the first metatarsophalangeal joint, the synovial fluid containing intracellular negatively birefringent needle shaped crystals.

\section{UNDIFFERENTIATED OLIGOARTHRITIS}

The remaining 34 patients were diagnosed as having undifferentiated oligoarthritis (table 2). The median duration of the arthritis was seven weeks (interquartile range 3-67 weeks). In 15 (44\%) patients the arthritis was continuous and in $19(56 \%)$, it was episodic. Seven $(21 \%)$ had a family history of arthritis. The pattern of arthritis in men and women was lower limb, pauciarticular arthritis with a predilection for the knee and ankle joints. It was an oligoarthritis, with $15(44 \%)$ patients presenting with monarthritis. The median number of joints affected was one (interquartile range one to two) and only four $(12 \%)$ patients had a polyarthritis with four or more joints affected. This was the first episode of arthritis in $14(41 \%)$ patients and the second in nine $(26 \%)$ patients. A history suggestive of urethral discharge was found in $30 \%$ of male patients, though whether this was sterile or infective in origin could not be determined retrospectively. There was no history of urethral or vaginal discharge in the women. Preceding diarrhoea occurred in 15 (44\%) patients.

Arthritis was the only feature in 20 (59\%), patients and in $11(32 \%)$ patients there was an associated enthesitis. The remaining three (9\%) patients included one with enthesitis alone, one with sacroiliitis alone, and one patient with symptoms but no objective signs. The joints affected were the knee in $18(53 \%)$, ankle, subtalar, or midtarsal in $14(41 \%)$, metacarpophalangeal or interphalangeal of the fingers in six $(17 \%)$, metatarsophalangeal or interphalangeal of the toes in five (15\%), wrist in five

Table 2 Features in the summary of the undifferentiated oligoarthritis group

\begin{tabular}{lc}
\hline Feature & Number (\%) of patients \\
\hline $\begin{array}{l}\text { Arthritis only } \\
\text { Arthritis and enthesitis }\end{array}$ & $20(59)$ \\
$\begin{array}{l}\text { Sacroiliitis (clinical) } \\
\text { Mucocutaneous features }\end{array}$ & $5(32)$ \\
$\begin{array}{l}\text { Previous urethritis } \\
\text { Previous diarrhoea }\end{array}$ & $5(15)$ \\
$\begin{array}{l}\text { Median (interquartile range) } \\
\text { of joints affected }\end{array}$ & $15(41)$ \\
$\begin{array}{l}\text { Joints most often affected } \\
\text { Knee }\end{array}$ & $1(1-2)$ \\
$\begin{array}{l}\text { Ankle/subtalar/midtarsal } \\
\text { Metatarsophalangeal/interphalangeal } \\
\text { foot }\end{array}$ & $18(53)$ \\
\hline
\end{tabular}

(15\%), hip in three $(9 \%)$, shoulder in three $(9 \%)$, and elbow in two $(6 \%)$ patients. No patient had a pattern of arthritis consistent with rheumatic fever or evidence of cardiac valve dysfunction on clinical examination.

Enthesitis on clinical examination was present in seven $(64 \%)$ women and five $(22 \%)$ men, occurring at the plantar aponeurosis insertion into the calcaneum in eight, trochanteric insertion of gluteus medius in seven, Achilles tendon insertion into the calcaneum in three, quadriceps insertion at the upper pole of the patella in one, the iliac crest in one, and the patella tendon insertion into the tibial tubercle in one. Tenosynovitis was present in three (9\%) patients, and rotator cuff tendinitis in two $(6 \%)$.

Axial skeleton disease was determined by pain on movement or restriction of movement, particularly lateral flexion. ${ }^{14}$ Seven $(20 \%)$ patients had disease of the lumbar spine, two the thoracic, and one the cervical spine. Pain in the sacroiliac joints when stressed or on local palpation was present in five patients. Overall spinal flexibility measured by $\mathrm{C} 7$ to iliac crest distraction ${ }^{15}$ and lumbar spinal movement measured by modified Schober index ${ }^{16}$ was the same as for age matched normal white subjects. ${ }^{16}{ }^{17}$ All patients with restricted lumbar movement were in the lower quartile of this measurement.

Mucocutaneous features were present in five $(15 \%)$ men and consisted of balanitis in three, conjunctivitis in one, mouth ulceration in one, and urethritis in one. There were no mucocutaneous features in the women.

The peripheral blood white cell count was increased in six (18\%) subjects and the median ESR was $26 \mathrm{~mm} /$ hour (interquartile range 12-38). Wuchereria bancrofti microfilaraemia was not detected in any subject.

Synovial fluid analysis from 17 patients with undifferentiated oligoarthritis showed an inflammatory white cell count of $2 \times 10^{9} / 1$ or more in $10(59 \%)$, and $10 \times 10^{9} / 1$ or more in five $(29 \%)$ patients, with an overall median value of $3 \times 10^{9} / 1$. The median percentage of polymorphonuclear leucocytes was $61 \%$ (interquartile range 41-83).

IgM and IgA rheumatoid factors were not significantly different from area matched controls. Antinuclear antibodies were present in one patient (titre 1/160 with a speckled pattern).

An increased titre of antibodies to streptolysin $O(\geqslant 320)$, was present in one patient and three controls. An increased titre of antibodies to deoxyribonuclease $B(\geqslant 640)$ was present in 18 patients and four controls $\left(\chi^{2} ; \quad p<0.01\right)$. Thirty eight per cent of patients with gonococcal arthritis and $44 \%$ of patients with an oligoarthritis had an increased titre of antibodies to deoxyribonuclease $B$ (NS).

The HLA-B27 phenotype was positive in $31 \%$ of the patients with undifferentiated oligoarthritis compared with $14 \%$ of controls $(t=1.824 ; p=0.072)$.

\section{Discussion}

Undifferentiated oligoarthritis is a major source of diagnostic confusion in developing tropical 
countries, where septic arthritis is often suspected, yet microbiological facilities are limited. The incidence of culture positive septic arthritis varies with the location of the study and microbiological facilities, from $0 \%$ in Port Moresby ${ }^{56}$ on the south coast, to $7 \%$ in the highlands, and $14 \%$ in Madang ${ }^{5}$ on the north coast. Our results showed $19 \%$ of patients with arthritis being assessed in the Papua New Guinea highlands to have a septic cause, all due to $N$ gonorrhoeae. This result is similar to that from a study of acute tropical polyarthritis in Zimbabwe, in which $20 \%$ had gonococcal arthritis. ${ }^{18}$ It is also in agreement with Scrimgeour $e t$ $a l^{4}$ who found gonococcal arthritis to be the most common diagnosable disease. Despite having higher synovial fluid white cell counts and a lower frequency of enthesopathy and mucocutaneous features, patients with gonococcal arthritis could only be distinguished by positive synovial fluid culture.

The delay in growth of gonococcus on culture could be explained by the widespread use of penicillin and chloramphenicol by clinics, rural health centres, and aid posts leading to partially treated gonococcal arthritis. Synovial fluid cultures are positive in as few as $25-50 \%$ of patients with disseminated gonococcal infection, ${ }^{19}$ and although this makes our prevalence value likely to be an underestimate, previous reports may have allowed insufficient incubation time. The culture system used here is costly for developing countries, but it performed much better than direct plating of synovial fluid, which did not detect $N$ gonorrhoeae in the same specimens. This may be due to the larger volume of synovial fluid $(4 \mathrm{ml})$ with a consequently larger inoculum of organisms. The broth used, and the closed culture system with daily subculture by inversion of the bottle onto chocolate agar, may be more conducive to the isolation of small numbers of fastidious organisms such as $N$ gonorrhoeae. This could be due to lysis of cells that contain gonococci or neutralisation of inhibiting factors in the synovial fluid. Future studies of tropical arthritis may consider using this method. Bedside inoculation of plates using a candle jar extinction method may be the cheapest alternative. The high number of PPNG isolates (50\%) means that penicillin can no longer be considered to be an adequate empirical treatment for presumed gonococcal septic arthritis in Papua New Guinea. Initial parenteral treatment would ideally be ceftriaxone. ${ }^{20}$ Cost constraints in developing countries mean that inferior parenteral treatments (for example, chloramphenicol) or less suitable treatments by mouth (for example, amoxycillin plus clavulanic acid, fluoroquinolones) will probably be used instead.

Most patients presenting with musculoskeletal symptoms in the highlands of Papua New Guinea had a pattern of arthritis consistent with reactive arthritis as has been previously suggested. ${ }^{5}$ Pathogens implicated in the aetiology of reactive arthritis which are common in Papua New Guinea include salmonella and shigella species, as well as Chlamydia trachomatis. ${ }^{21}$ Lower limb asymmetrical oligoarthritis accompanied by tendinitis or sacroiliitis is characteristic of the seronegative spondyloarthropathies. The additional presence of preceding infection or mucocutaneous features is particularly indicative of reactive arthritis. ${ }^{22}$

Arthritis and extra-articular disease, including mucocutaneous features and enthesopathy, or preceding diarrhoea was present in 21/34 (62\%) of patients and a history suggestive of urethral discharge in $30 \%$ of the male patients; these are consistent with reactive arthritis. Axial spinal disease was clinically present in a further $12 \%$ of patients. The inability to determine a history of preceding enteric or genital infection in the remainder does not negate the diagnosis of reactive arthritis, as the infection may be asymptomatic. ${ }^{23} 24$ It is also acknowledged that an accurate and reliable history is often difficult to obtain in societies such as those in the Papua New Guinea highlands, even when communication (by interpreter, or Melanesian tok pisin) is optimal. The outcome of the arthritis was not determined because in a predominantly rural indigenous migratory population in a country such as Papua New Guinea follow up studies are notoriously difficult to perform.

The class I molecule HLA-B27 is increased in the seronegative spondyloarthropathies and has been implicated in the aetiopathogenesis of reactive arthritis. ${ }^{25}$ Previous studies of undifferentiated polyarthritis in Papua New Guinea found the prevalence of HLA-B27 ranging from 30 to $70 \%, 52627$ compared with control rates of $5-17 \%{ }^{5} 28$ The lower than expected prevalence of $31 \%$ HLA-B27 in this study may imply that in this community the part played by environmental factors in aetiology varies over time.

Viral and parasitic infections have been considered as causes of tropical polyarthritis ${ }^{428}$ but in this series no patient had the clinical or serological evidence of Ross River virus arthritis or $W$ bancrofti microfilaraemia. The study area has little filariasis, so that the lack of positive results for $W$ bancrofti is not surprising. Since the study of Salfield, ${ }^{29}$ it has been difficult to clarify the role of filariasis in tropical polyarthritis in Papua New Guinea, owing to variation in endemicity throughout the country. Initial testing for Ross River virus by ELISA yielded eight positive results for Ross River virus IgM. These could not be confirmed by class capture IgM and were most probably false positives. A similar problem with Ross River virus ELISA testing has been found before with serum samples from subjects in Papua New Guinea (A Cunningham, unpublished data). This further shows the difficulties encountered with such antibody tests.

The results for antibodies to streptolysin $\mathrm{O}$ and antibodies to deoxyribonuclease $B$ are difficult to relate to tropical polyarthritis in a community where streptococcal skin infections are common. The levels of antibodies to deoxyribonuclease $B$ were increased in the group with arthritis but were equally divided between the groups with gonococcal and oligoarthritis. The significance of the results to the aetiopathogenesis of the arthritis is unclear but it is probably a non-specific increase associated with other infections or inflammation. 
Rheumatoid arthritis has been described in Papua New Guinea, ${ }^{4}$ though its prevalence is low, ${ }^{30}$ and we did not find any clinical or serological evidence of rheumatoid arthritis.

Tropical polyarthritis as it occurs in the highlands of Papua New Guinea is not a distinct homogeneous entity, but includes septic arthritis and reactive arthritis. It is a term that should no longer be used as it does not refer to a specific arthritis, and includes arthritides not specific to tropical countries. The diagnosis of reactive arthritis is based on history and physical findings related to enteric and genital infection, the pattern of arthritis and extra-articular disease of mucous membranes and entheses.

This study supports investigations into the aetiology in a patient with polyarthritis and confirms that despite limited laboratory resources, septic arthritis can be identified. Sexually transmitted disease and enteric infections are implicated in this study as major contributing factors to polyarthritis and resultant morbidity affecting young men and women in the highlands of Papua New Guinea. Reduction in infection by public health measures, combined with appropriate antibiotic treatment of infections would be the initial approach to help reduce the morbidity from arthritis.

We express our thanks to Associate Professor A Cunningham department of microbiology, Westmead Hospital for the Ross River virus testing using the class capture assay, Dr M P Alpers, Director, PNGIMR for facilitating the study, and Mr R Spark, Laboratory Manager, PNGIMR. Dr J R L Forsyth (University of Melbourne) supplied the set of 12 monoclonal antibodies to gonococcal outer membrane epitopes.

1 Anonymous. Acute tropical polyarthropathy: homogeneous entity or diagnostic scrap-heap? [editorial]. Lancet 1988; 627-8.

2 Jeremy R, Rhodes F A, Sharp J T, Rawls W E. Clinical and laboratory studies of a distinctive type of arthritis observed laboratory studies of a distinctive type of arthritis

3 Maddocks I. Reiter's disease in Port Moresby, Papua. Br $\mathcal{J}$ Vener Dis 1967; 43: 280-3.

4 Scrimgeour E M, Matz L R, Aaskov J G. A study of arthritis in Papua New Guinea. Aust $N Z \mathcal{F}$ Med 1987; 17: $51-4$

5 Richens J, Hudson B, Naraqi S, Lloyd A. A study of arthritis in Papua New Guinea. Aust N Z F Med 1988; 18: 807-8.

6 Lloyd A, Mavo B, Cunningham A, Naraqi S. Tropical arthritis in Papua New Guinea: a reactive arthritis. Med $\mathcal{f}$ Aust 1990; 152: 186-9.

7 Clunie G P R, Koki G, Prasad M L, Richens J E, Bhatia K Keat A. HLA-B27, arthritis and spondylitis in an isolated community in Papua New Guinea. Br 7 Rheumatol 1990; 29: 97-100

8 Tapsall J W, Cheng J K. Rapid identification of pathogenic species of Neisseria by carbohydrate degradation tests: importance of glucose in media for preparation of inocula. Br F Vener Dis 1981; 57: 249-52.

9 Australian gonococcal surveillance programme. Penicillin sensitivity of gonococci in Australia: development of Australian gonococcal surveillance programme. $B r \mathcal{F} V e n e r$ Dis 1984; 60: 226-30.

$10 \mathrm{La}$ Scolea L J, Young F E. Development of a defined medium for Neisseria gonorrhoeae. Appl Microbiol 1974; 28: $\%-6$

11 Freedman D O, Nutman T B. Filariasis. In: Balows A, Hausler W J, Ohashi M, Turano A, eds. Laboratory diagnosis of infectious diseases. Principles and practice. Vol. 1 . New York: Springer Verlag, 1988: 863-71.

12 Carter I, Smythe L, Fraser J, et al. Detection of Ross River virus immunoglobulin $M$ antibodies by enzyme-linked immunosorbent assay using antibody class capture and comparison with other methods. Pathology 1985; 17: 503-8.

13 Bhatia K, Gorogo M, Koki G. HLA-A, B, C and DR antigens in Asaro speakers of Papua New Guinea. Hum Immunol 1984; 9: 189-200.

14 Tomlinson $M$ J, Barefoot J, Dixon A St J. Intensive inpatient physiotherapy courses improve movement and posture in ankylosing spondylitis. Physiotherapy 1986; 72: $238-40$.

15 Joint motion. Method of measuring and recording. fournal of the American Academy of Orthopaedic Surgeons 1965: 1-87.

16 Macrae I F, Wright V. Measurement of back movement. Ann Rheum Dis 1969; 28: 584-9.

17 Moll J M H, Wright V. Normal range of spinal mobility. An objective clinical study Ann Rheum Dis 1971; 30: 381-6.

18 Stein C M, Hanly M G. Acute tropical polyarthritis in Zimbabwe: a prospective search for a gonococcal aetiology. Ann Rheum Dis 1987; 46: 912-4.

19 Goldenberg D L. Infectious arthritis. In: Schumacher H R, ed. Primer on the rheumatic diseases. 9th ed. Atlanta GA Arthritis Foundation, 1988: 181-5.

201989 Sexually transmitted diseases guidelines $M M W R \quad 1989$ 38: $S-8,24-25$.

21 Wesselingh S, Hallsworth P, McDonald P J, Richens J E. Chlamydial infection in Papua New Guinea. Trans $R$ So Trop Med Hyg 1988; 82: 333 .

22 Keat A. Reiter's syndrome and reactive arthritis in perspective. N Engl F Med 1983; 309: 1606-15.

23 Keat A, Thomas B, Hughes R, Taylor-Robinson D. Chlamydia trachomatis in reactive arthritis. Rheumatol Int 1989; 9 197-200.

24 Toivanen A, Tertti $R$, Lahesmaa-Rantala $R$, Stahlberg $T$ Granfors K. Factors associated with the development of reactive arthritis. $\mathrm{Br} \mathcal{F}$ Rheumatol 1988; 27 (suppl II): 46-51.

25 Aho $\mathrm{K}$, Ahvonew $\mathrm{P}$, Lassus A, Sievers $\mathrm{K}$, Tiilikainew A. HLA-B27 in reactive arthritis. A study of yersinia arthritis HLA-B27 in reactive arthritis. A study of yersinia arth

26 Richens J E, Prasad M L, Bhatia K, Tung M. Arthritis and HLA-B27 in Papua New Guinea. Br Med F Clin Res 1986; 293: 1209.

27 Vaterlaws A, Wolnizer M, Doran T, Bashir H, Bhatia K. HLA and nonspecific polyarthritis in the highlands of Papua New Guinea. Papua New Guinea Medical fournal 1989; 32: 263-8

28 Serieantson S W. HLA and disease in Oceania. Papua New Guinea Medical foumal 1989; 32: 241-9.

29 Salfield S. Filarial arthritis in the Sepik district of Papua New Guinea. Fed f Aust 1975; 1: 264-7.

30 Dewdney J C H, MacLennan R. Rheumatoid arthritis in Maprik, Sepik district, New Guinea. Med f Aust 1966; 835-7. 